\title{
The caesarean section rate: Much ado about nothing?
}

In 1985, the World Health Organization (WHO) recommended that population-based caesarean section (CS) rates should be between 10 and $15 \% .{ }^{[1]}$ This recommendation was based on expert consensus opinion in response to what was considered an unjustified increase in the CS rate. ${ }^{[1]}$ Since then, more recent reports of international CS rates have vared between 6 and 27\%, depending on the population studied. ${ }^{[2]}$ Ecological studies in the last decade have not been able to determine the 'perfect' CS rate, with some studies estimating that it is below, while others reporting it to be above, the hallowed $15 \%$. ${ }^{[2,3]}$

It is undeniable that CSs are lifesaving procedures when used appropriately. Underuse leads to an increase in maternal and perinatal morbidity and mortality. ${ }^{[4]}$ An excessively high CS rate is, by definition, unnecessary. Increasing the CS rate increases the rate of complications such as operative bleeding and short- and longterm neonatal adverse outcomes, and affects future pregnancies through placental attachment disorders. ${ }^{[5,6]}$ The increase in the CS rate in low- and middle-income countries has been large, although the absolute rate may still remain below $15 \%{ }^{[2]}$

So, what drives the increase in the CS rate? Medical or obstetric indications are justifiable, and should result in improved maternal and perinatal outcomes. However, various studies have demonstrated that the increase, especially during the last two decades, has not resulted in improved outcomes. ${ }^{[1-3,7]}$ Therefore there must be other, non-medical, factors at play. Professional practice, an increase in obstetric-related malpractice, and economic, organisational, social and cultural preferences have played a major role in driving the CS rate. ${ }^{[2,8,9]}$

Current strategies to control or reduce the CS rate have focused on medically-directed strategies, via adherence to protocols, CS indication analysis (as described in an article in this journal ${ }^{[10]}$ ) and peer review. These techniques have, despite good intentions, largely been unsuccessful thus far. Commitment to women-centred care is recommended to optimise the CS rate. This approach encourages a multidisciplinary approach, with collaborative respectful care. This model includes emphasising continuous labour support, adequate pain control in labour and the correct timing and indication of induction of labour. ${ }^{[9]}$ Scaling up midwifery-led services, especially in resource-constrained environments, reduces adverse outcomes and can alter the CS rate. ${ }^{[1]}$

However, mother-baby-friendly hospital care, education and birth preparation classes, and the introduction of standardised protocols have thus far not had a significant impact on controlling or reducing the CS rate. The only significant reduction has been demonstrated when the appropriate use of CS and the CS rate have influenced reimbursement for doctors and hospitals. ${ }^{[11,12]}$

In South Africa (SA), we have seen a decline in maternal mortality in the last triennium. This success is largely attributed to the decline in non-pregnancy related infections; HIV is being managed more effectively. ${ }^{[5]}$ However, the CS rate remains a concern, with an increase in case fatality rates for excessive bleeding associated with CS. ${ }^{[5]}$ In SA, the burden related to CS is bifocal: (i) the increasing CS rate in the private sector; and (ii) an increase in haemorrhage related to CS in the public sector.

In $\mathrm{SA}$, the introduction of the BetterObs training programme and the Essential Steps in the Management of Medical Emergencies
(ESMOE) programme to upskill practitioners has not completely addressed CS-related complications and their consequences. The question remains as to whether women requiring CS are actually receiving the appropriate care. ${ }^{[5]}$

Thus far, the focus has largely been on the CS rate itself. It is still believed that a rate of $10-15 \%$ is desirable, despite the fact that some studies have shown that a rate of up to $19 \%$ is required to optimise benefits and risk to mother and neonate. However, by focusing on the CS rate alone, we are not addressing some of the important underlying questions.

It may be time to move away from the rate, and to focus on the pregnancy and a case-by-case outcome. The question remains: are all the women who require CS being delivered by CS? The current focus on CS rates does not answer this question. In addition, the quoted rate of $10-15 \%$ is recommended for populations - it is not practice- or institution-based. The case load, type of practice and level of health facility needs to be taken into consideration to determine the optimal CS rate.

The Robson 10 classification ${ }^{[1]}$ is a standardised system that can be used in any labour and delivery setting to categorise the indication for CS. However, the reliability of this system will depend on the veracity of the practitioner, especially in the event of non-medically indicated CS. While this system has its flaws and loopholes, it moves the focus away from the CS rate and rather to the indication for CS, and takes the obstetric management of individual cases into consideration, which is more relevant.

The International Federation of Gynecology and Obstetrics (FIGO) currently recommends the following strategies to lower CS rates: ${ }^{[1]}$

- Review delivery fees for deliveries in private practice.

- Hospitals and private practices should publish annual CS rates. Financing should be partly based on risk-adjusted CS rates. However, this strategy requires clinical insight into the obstetric management of cases, and consideration of the level of care e.g. tertiary academic hospitals should be expected to have higher CS rates and more morbidity, as these institutions care for the most complicated high-risk cases.

- Hospitals/practices should use uniform classification systems for CS, e.g. Robson 10, the WHO classification.

- Women must be properly informed about the benefits and risks of CS. Uniform information with uniform relevant evidence-based information should be offered to all pregnant women, regardless of healthcare setting.

- Money generated from reducing CS should be invested in practical skills training, pain management and better labour and delivery care.

- Rural areas require very specific attention, as these seems to be where access to CS and the appropriate operative skills are insufficient. Adequate skills, healthcare facilities and labour and delivery care are essential in these areas.

The focus needs to move from the CS rate to women-centred care in labour and delivery, to optimise the birth experience and outcomes. This, although a long process, will result in the appropriate CS rate for each centre, with improved maternal and perinatal outcomes associated with justifiable risks. 
Sumaiya Adam, MB ChB, FCOG (SA), MMed (Obstetr Gynaecol), Cert Maternal Fetal Med (SA), PhD (Obstetr Gynaecol)

Department of Obstetrics and Gynaecology, Steve Biko Academic Hospital, University of Pretoria, South Africa sumaiya.adam@up.ac.za

S Afr J Obs Gynae 2018;24(3): 68. DOI:10.7196/SAJOG.2018.v24i3.1524

1. Betran AP, Torloni MR, Zhang JJ, et al. WHO statement on caesarean section rates. BJOG 2016;123: 667-670. https://doi.org/10.1111/1471-0528.13526

2. Betran AP, Jianfeng $\mathrm{Y}$, Moller $\mathrm{AB}$, et al. The increasing trend in caesarean section rates: Global, regional and national estimates 1990 - 2014. PloSONE 2016;11(2):e0148343 . https://doi. org/10.1371/journal.pone. 0148343

3. Ye J, Zhang J, Mikolajczyk, et al. Association between rates of caesarean section and maternal and neonatal mortality in the 21st century: A worldwide population-based ecological study with longitudinal data. BJOG 2016;123(5):745-753. https://doi.org/10.1111/1471-0528.13592
4. Betran AP, Temmerman M, Kingdon C, et al. Interventions to reduce unnecessary caesarean sections in healthy women and babies. Lancet 2018;392(10155):1358-1368. https://doi.org/10.1016/ s0140-6736(18)31927-5

5. Moodley J, Fawcus S, Pattinson R. Improvements in maternal mortality in South Africa. S Afr Med J 2018;108(3 Suppl 1):S4-S8. https://doi.org/10.7196/SAMJ.2018.v108i3.12770

6. Sandall J, Tribe RM, Avery L, et al. Short-term and long-term effects of caesarean section on the health of women and children. Lancet 2018;392(10155):1349-1357. https://doi.org/10.1016/s01406736(18)31930-5

7. Molina G, Weiser TG, Lipsitz SR, et al. Relationship Between Caesarean section Rate and Maternal and Neonatal Mortality. JAMA 2015;314(1):2263-2270.

8. Wiklund I, Malata AM, Cheung NF, Cadee F. Appropriate use of caesarean section globally requires a different approach. Lancet 2018;392(10155):1288-1289. https://doi.org/1016/S01406736(18) $31927-5$

9. Renfrew MJ, McFadden A, Bastos MH, et al. Midwifery and quality care: findings from a new evidence-informed framework for maternal and newborn care. Lancet 2004;384(9948):1129-1145 https://doi.org/10.1016/S0140-6736(14)60789-3

10. Guidozzi DF, Branch S, Chauke L. Maternal and fetal outcomes following delivery in a tertiary hospital in Johannesburg, South Africa. S Afr J Obstet Gynaecol 2018;24(3):xx-xx. https://do org/10.7196/SAJOG.2018.v24i3.1396

11. Visser GHA, Ayres-de-Campos D, Barnea ER, et al. FIGO position paper: How to stop the

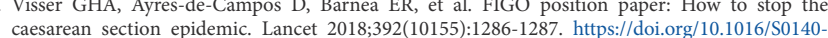
caesarean section

12. The Lancet. Editorial. Stemming the global caesarean section epidemic. Lancet 2018;392(10155):1279. https://doi.org/10.1016/s0140-6736(18)32394-8 